\title{
Camera Model and Calibration Procedure for Oblique-Viewing Endoscope
}

\author{
Tetsuzo Yamaguchi ${ }^{1}$, Masahiko Nakamoto $^{1}$, Yoshinobu Sato ${ }^{1}$, \\ Yoshikazu Nakajima ${ }^{1}$, Kozo Konishi ${ }^{3}$, Makoto Hashizume ${ }^{3}$, Takashi Nishii ${ }^{2}$, \\ Nobuhiko Sugano ${ }^{2}$, Hideki Yoshikawa ${ }^{2}$, Kazuo Yonenobu ${ }^{4}$, and \\ Shinichi Tamura ${ }^{1}$ \\ 1 Division of Interdisciplinary Image Analysis, \\ 2 Department of Orthopaedic Surgery, Osaka University Graduate School of Medicine \\ 3 Graduate School of Medical Sciences, Kyushu University \\ 4 Osaka Minami National Hospital
}

\begin{abstract}
Oblique-viewing endoscopes (oblique scopes) are widely used medically. It is essential for certain procedures such as laparoscopy, arthroscopy, and sinus endoscopy. In an oblique scope, its viewing directions are changeable by rotating the scope cylinder. Although a camera calibration method is necessary to apply augmented reality technologies to oblique endoscopic procedures, no method for oblique scope calibration has been developed yet. In the present paper, we formulate a camera model and a calibration procedure for oblique scopes. In the calibration procedure, Tsai's calibration is performed at zero-rotation of the scope cylinder, and then the variation of the external camera parameters corresponding to the rotation of the scope cylinder is modeled and estimated as a function of the rotation angle. Accurate estimation of the rotational axis is included in the procedure. The precision of this estimation was demonstrated to have a significant effect on the overall calibration accuracy in the experimental evaluation especially with large rotation angles. The projection error in the image plane was around two pixels. The proposed method was shown to be clinically applicable.
\end{abstract}

\section{Introduction}

As a procedure with minimal invasion, endoscopic surgery is becoming common in recent years. When rigid scopes are used, the movements of the endoscope can be tracked by a 3D position sensor attached to the endoscope body outside the cavity. Since endoscopic surgery is essentially a monitor-based operation, conventional monitor-based augmented reality (AR) technologies may be combined straightforwardly with rigid scope tracking without any special display devises or 3D glasses [1, 2], 3]. Several AR systems for rigid scopes including microscopes have already been used clinically. These systems superimpose virtual and real endoscopic images accurately. Previous studies have investigated additional parameters for endoscopic camera calibration other than those in the conventional Tsai's method [4], and the correction of distortion caused by wide-angle lenses 
is often incorporated [5], [6], [7], 8]. Moreover, the zoom and focus parameters for microscopes have been examined for microscopic camera calibration [9]. The zoom and focus parameters are dynamically changeable during the endoscopic procedures while the distortion parameters are static.

There are two types of rigid endoscopes, a forward-viewing endoscope ("forward scope") and an oblique-viewing or side-viewing endoscope ("oblique scope"). Because the range of movement of the scope cylinder is considerably restricted due to fixed entry point and constriction of the cavities, it is often difficult to observe a target object from desired viewing directions using forward scopes in which the viewing direction is aligned and fixed to the axis of the scope cylinder. By contrast, in oblique scopes, the viewing direction has a tilt (for example, of 30 degrees) from the axis direction of the scope cylinder and is changeable by rotating the scope cylinder around the cylinder axis. Thus, oblique scopes are more advantageous than forward scopes due to their much wider variety of viewing directions attained by the rotation of the scope cylinder. To our knowledge, no camera model of oblique scopes to date has been developed in spite of the fact that oblique scopes are widely used in clinical practice and they are even essential for certain procedures such as laparoscopy, arthroscopy, and sinus endoscopy.

In this paper, we propose a camera model for oblique scopes as an extension of Tsai's model, and develop its calibration method. While lens distortion, zoom, and focus parameters are involved in modification of the internal parameters in Tsai's camera model, the rotation parameter of the scope cylinder in oblique scopes is related to the external parameters. This rotation parameter is dynamically changeable during scope operation, similarly to zoom and focus parameters. We formulate the camera model for oblique scopes that relates the rotation parameter of the scope cylinder to the external parameters in Tsai's camera model. Further, we develop a calibration method for estimating the parameters involved in the process.

\section{Methods}

\subsection{Overview of Oblique-Viewing Endoscope}

The features of an oblique scope in comparison with a forward scope are illustrated in Fig. 1. A rigid scope is characterized by its rigid scope cylinder. The difference in the appearance between oblique and forward scopes is observed at the tips of their scope cylinders. As shown in Fig. 1(a), the viewing direction of a forward endoscope is aligned to the axis of the scope cylinder, while an oblique scope has a tilt from the axis direction and its viewing direction is changeable by rotating the scope cylinder around the axis, Fig. 1(c) and (d) present images of a world map on a cylindrical inner wall acquired by real forward and oblique scopes, respectively, at their positions shown in Fig 1(b). As demonstrated in Fig. 1(d), oblique endoscopic images are changed by rotating the scope cylinder, and each region of the world map on the inner wall is much more clearly observed than that obtained through forward scope. 
We attach additional apparatus for measuring the pose of the camera head and the rotation of the scope cylinder as follows. A rigid body with optical markers is attached to the scope body for measuring its $6 \mathrm{D}$ pose parameters using an optical tracker, and the rotary encoder is geared to the scope cylinder for measuring its rotation parameter. The rigid body defines the world coordinate system in the camera model described in the following subsections.

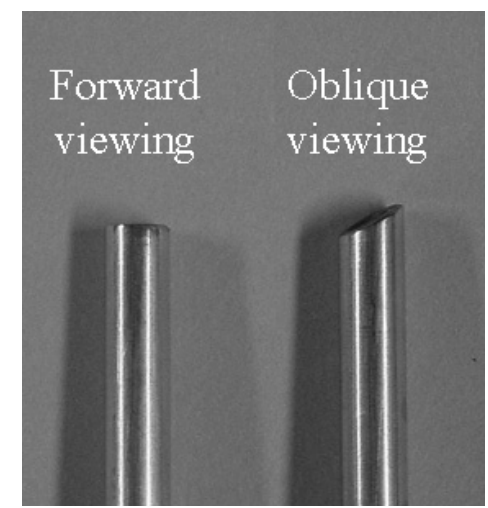

(a)

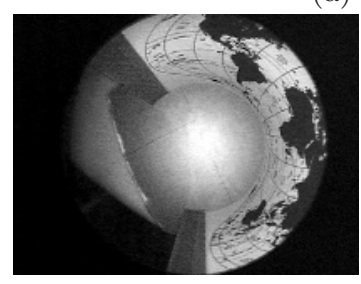

(c)

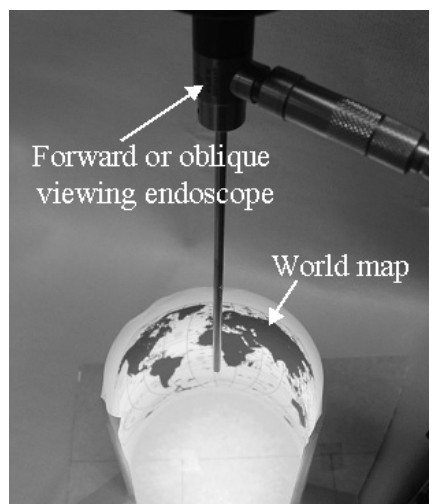

(b)
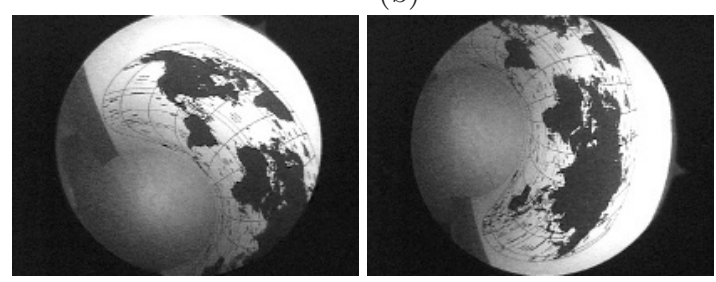

(d)

Fig. 1. Comparisons between forward-viewing and oblique-viewing endoscopes. (a) Comparison of the tip of scope cylinder. (b) Set-up for acquisition of endoscopic images. (c) Forward-viewing endoscopic image. (d) Oblique-viewing endoscopic images with $\theta=0^{\circ}$ (left) and $60^{\circ}$ (right).

\subsection{Tsai's Camera Model}

In Tsai's camera model, the camera parameters are classified into external and internal parameters. 4]. The external parameters determine the transformation from the world coordinate system $S_{w}$ to the camera coordinate system $S_{c}$. The internal parameters determine the projection of $3 \mathrm{D}$ points in the camera coordinate system onto the $2 \mathrm{D}$ image $I_{c}$. The camera parameters inherent in oblique scopes are mainly related to the external parameters. Let a $3 \mathrm{D}$ point in $S_{w}$ be $\mathbf{p}_{w}=\left(x_{w}, y_{w}, z_{w}, 1\right) \in S_{w}$, and that in $S_{c}$ be $\mathbf{p}_{c} \in S_{c}$. Given rigid transformation matrix $T: S_{w} \mapsto S_{c}$, we have

$$
\mathbf{p}_{c}=T \mathbf{p}_{w}
$$


Let $I$ be the image coordinate system, and $\mathbf{i}={ }^{t}(u, v, 1) \in I$ be projected image of $\mathbf{p}_{c}$ onto $I$. Given image projection matrix $M$, we have

$$
s \mathbf{i}=M \mathbf{p}_{c}=M T \mathbf{p}_{w},
$$

where $s$ is a scalar value and $s=z_{c}$ since the $z_{c}$-axis is set as the optical axis. $T$ in Eq. (1) and $M$ in Eq. (2) include the external and internal camera parameters, respectively.

\subsection{Simple Camera Model for Idealized Oblique-Viewing Endoscope}

In the camera model for oblique scopes, rigid transformation matrix $T$ representing the external parameters is described as a function of the rotation angle $\theta$ of the scope cylinder, and thus represented as $T(\theta)$. Fig. 2(a) shows a schematic diagram of the idealized camera model. The right panel of Fig. 2(a) illustrates the relation between the camera coordinate system $S_{c}$ and the rotation axis $l_{s}$ of the scope cylinder in the camera model of an idealized oblique scope. The origin of $S_{c}$ ideally passes through $l_{s}$. The $z$-axis of $S_{c}$, i.e. the viewing direction, is rotated around $l_{s}$ connected with the rotation $\theta$ of the scope cylinder while the angle between $l_{s}$ and the $z$-axis of $S_{c}$ remains fixed at the oblique viewing angle $\phi_{0}$ of the scope. Here, it is necessary to carefully model the fact that due to the rotation of the scope cylinder, only the lens system rotates while the camera head, i.e. the imaging plane, remains fixed irrespective of the rotation. Therefore, in our mathematical representation, the rotation $\theta$ around $l_{s}$ of the coordinate system $S_{c}$ is executed first (right panel of Fig. 2(a)), and then, the $x y$-plane of $S_{c}$ is rotated by $-\theta$, i.e. rotated inversely (center panel of Fig. $2(\mathrm{a})$ ). Let $l_{h}$ be the axis of the second rotation. Ideally, $l_{h}$ corresponds to the $z$-axis. The first mathematical rotation involves the movements of both the lens system and the imaging plane. Nevertheless, the rotation of the imaging plane should not occur. Because the rotation of the camera head around $l_{s}$ in the physical space virtually corresponds to the rotation of the imaging plane around the $z$-axis of $S_{c}\left(l_{h}\right)$, the incorrect rotation of the imaging plane is nullified by the second rotation of the $x y$-plane of $S_{c}$, because the horizontal and vertical axes of the imaging plane are aligned to the $x$ - and $y$-axes of $S_{c}$.

Let $T_{r}(\theta ; \mathbf{v}, \mathbf{x})$ or $T_{r}(\theta ; l)$ be transformation matrix representing the rotations $\theta$ around the axis $l$ defined by point $\mathbf{x}$ and direction $\mathbf{v}$. Let $\mathbf{o}_{c}$ be the origin of $S_{c}$, and $\mathbf{m}$ be the direction of $l_{s}$ (where $|\mathbf{m}|=1$ ). Let $\mathbf{z}_{c}(\theta)$ be the direction of the $z$ axis of $S_{c}$ when the rotation angle of the scope cylinder is $\theta$, that is, $S_{c}$ is rotated by $\theta$ around $l_{s}$. Let $T_{0}$ represent the transformation matrix from $S_{w}$ to $S_{c}$ when $\theta=0$, that is, the scope cylinder is at zero rotation. $T_{0}$ is rigid transformation representing the external parameters in Tsai's camera model when $\theta=0$. When the scope cylinder is rotated by $\theta$, rigid transformation matrix representing the external parameters in Tsai's camera model, $T(\theta)$, can be given by

$$
T(\theta)=T_{0} T_{r}\left(-\theta ; \mathbf{m}, \mathbf{o}_{c}\right) T_{r}\left(\theta ; \mathbf{z}_{c}(\theta), \mathbf{o}_{c}\right),
$$

where $T_{r}\left(-\theta ; \mathbf{m}, \mathbf{o}_{c}\right)$ and $T_{r}\left(\theta ; \mathbf{z}_{c}(\theta), \mathbf{o}_{c}\right)$ physically correspond to the rotations of the scope cylinder and the camera head, respectively. 


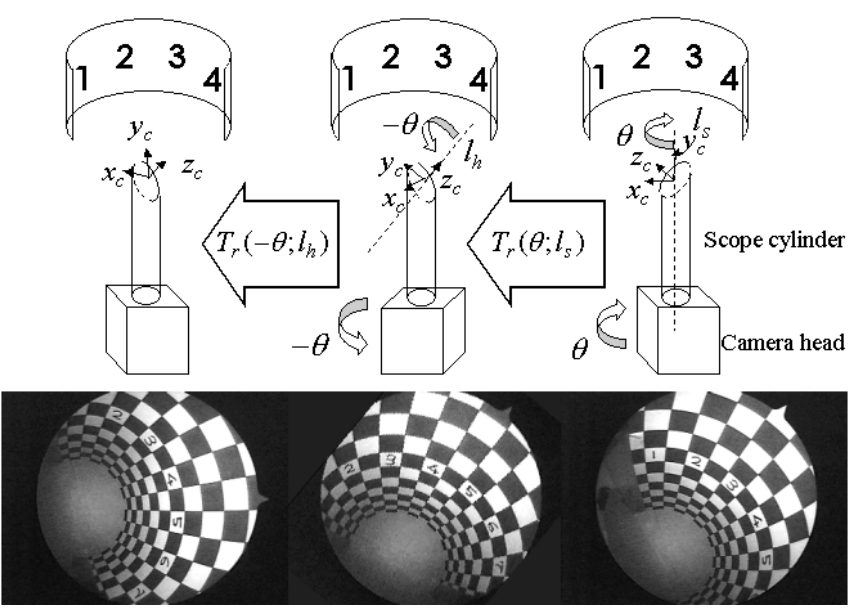

(a)
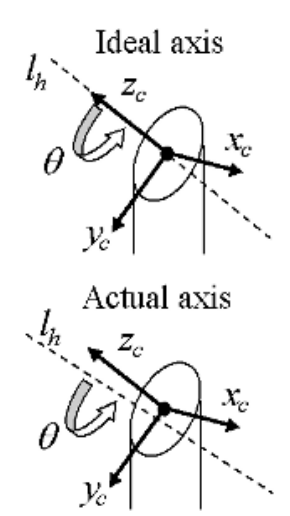

(b)

Fig. 2. Schematic diagram of camera model for oblique-viewing endoscope (a) Modeling of idealized oblique scope. (b) Modeling of rotational axis $l_{h}$ in idealized model (top) and general model (bottom).

\subsection{Extended Camera Model for General Oblique-Viewing Endoscope}

Modeling of actual oblique scopes needs to incorporate deviations from the idealized model for accurate calibration. In the idealized model, several ideal situations are assumed. The following two violations of the assumptions are considered to seriously affect the accuracy in the calibration of actual oblique scopes.

- The origin of $S_{c}$ does not pass through the axis $l_{s}$.

- In the second rotation, the rotation axis $l_{h}$ of the $x y$-plane of $S_{c}$, i.e. the imaging plane, is deviated from the $z$-axis of $S_{c}$ (Fig. 2(b)).

To address the first deviation, we simply assume that $l_{s}$ does not necessarily pass through $\mathbf{o}_{c}$ and $l_{s}$ is defined by point $\mathbf{q}$ and orientation $\mathbf{m}$. To tackle the second, we define $l_{h}$ as a function of rotation angle $\theta$ by $\mathbf{n}(\theta)$ and $\mathbf{r}(\theta)$ (where $|\mathbf{n}(\theta)|=1$ ). $l_{h}$ is the axis for the second rotation of the whole coordinate system of $S_{c}$, which rotates in correspondence to the first rotation of $S_{c}$. By incorporating the above additional parameters, $T(\theta)$ is rewritten by

$$
T(\theta)=T_{0} T_{r}(-\theta ; \mathbf{m}, \mathbf{q}) T_{r}(\theta ; \mathbf{n}(\theta), \mathbf{r}(\theta)),
$$

where $\mathbf{n}(\theta)$ and $\mathbf{r}(\theta)$ are written as

$$
\mathbf{n}(\theta)=T_{r}(-\theta ; \mathbf{m}, \mathbf{q}) \mathbf{n}_{0}, \quad \text { and } \quad \mathbf{r}(\theta)=T_{r}(-\theta ; \mathbf{m}, \mathbf{q}) \mathbf{r}_{0},
$$

in which $\mathbf{n}_{0}$ and $\mathbf{r}_{0}$ define the axis $l_{h}$ when $\theta=0$ (where $\left|\mathbf{n}_{0}\right|=1$ ). 


\subsection{Camera Calibration Procedure}

The camera calibration of oblique scopes involves the estimation of the following parameters.

- Rigid transformation $T_{0}$ from $S_{w}$ to $S_{c}$ at zero-rotation of the scope cylinder, i.e. the external camera parameters of Tsai's model at zero-rotation.

- Axis $l_{s}$, i.e. $\mathbf{m}$ and $\mathbf{q}$.

- Axis $l_{h}$ at zero-rotation of the scope cylinder, i.e. $\mathbf{n}_{0}$ and $\mathbf{r}_{0}$.

We assume that the determination of $S_{w}$ and the measurement of $\theta$ are practically possible. (In the experimental evaluation, we determined $S_{w}$ by measuring $6 \mathrm{D}$ pose parameters of the rigid body attached to the camera head using an optical tracker, and measured $\theta$ by the rotary encoder geared to the scope cylinder.)

The calibration procedure is as follows. Firstly, $T_{0}$ is estimated when the rotation of the scope cylinder is fixed at $\theta=0$. This step is performed based on the conventional Tsai's calibration procedure. Projection matrix $M$ representing the internal parameters is simultaneously estimated at this step. Secondly, $\mathbf{m}$ and $\mathbf{q}$ are estimated by $3 \mathrm{D}$ measurement of the rotation of the scope cylinder itself. And finally, $\mathbf{n}_{0}$ and $\mathbf{r}_{0}$ are estimated using oblique endoscopic images acquired at several rotations $\theta$ of the scope cylinder based on $T_{0}, \mathbf{m}$, and $\mathbf{q}$ estimated during the first and second steps. In the following, the second and third steps of the procedure are described in detail.

To estimate $\mathbf{m}$ and $\mathbf{q}$ defining the axis $l_{s}$, we attach an optical marker to a knob for rotating the scope cylinder (as shown in Fig. 3), and the 3D position of the optical marker is measured using an optical tracker during the rotation of the scope cylinder. The normal of the plane fitted to the measured 3D positions is regarded as $\mathbf{m}$, and the center of the circle fitted to their trajectory is regarded as $\mathbf{q}$. Let $\mathbf{p}_{s}^{i}$ be $i$-the measured 3D position, and $\mathbf{m}$ is estimated by minimizing $\sum_{i=1}^{m}\left\{\mathbf{m} \cdot \mathbf{p}_{s}^{i}+d\right\}^{2}$, where $\mathbf{m} \cdot \mathbf{p}_{s}^{i}$ denotes inner product, and $d$ is the distance from the origin of $S_{w}$ to the plane (which is not used in the camera model). Using estimated $\mathbf{m}, \mathbf{q}$ is estimated by minimizing $\sum_{i=1}^{m}\left\{\rho^{2}-\left|\mathbf{p}_{s}^{i}-\mathbf{q}\right|^{2}+\mathbf{m} \cdot \mathbf{p}_{s}^{i}-\mathbf{q}\right\}^{2}$, where $\rho$ is the radius of the circle (which is not used in the camera model).

To estimate $\mathbf{n}_{0}$ and $\mathbf{r}_{0}$ defining the axis $l_{h}$, we acquire endoscopic images at several rotation angles of the scope cylinder. Let $\mathbf{i}^{i j}$ be $i$-the $2 \mathrm{D}$ point in the endoscopic image acquired at rotation angle $\theta^{j}$, which is a projection of $3 \mathrm{D}$ position $\mathbf{p}_{h}^{j}$ in $S_{w}$. The relation between $\mathbf{i}^{i j}$ and $\mathbf{p}_{h}^{j}$ is described as $s \mathbf{i}^{i j}=$ $M T\left(\theta^{i}\right) \mathbf{p}_{h}^{j}$, where $s=z_{h}, M$ is the projection matrix including the internal parameters, and $T\left(\theta^{i}\right)$ is the rigid transformation matrix including the external parameters for oblique scopes. Because $M, T_{0}, \mathbf{m}$, and $\mathbf{r}$ are already estimated, only $\mathbf{n}_{0}$ and $\mathbf{r}_{0}$ are unknown in $T\left(\theta^{i}\right)$. Thus, the relation can be written as $\mathbf{i}^{i j}=\mathbf{i}\left(\mathbf{p}_{h}^{j}, \theta^{i} ; \mathbf{n}_{0}, \mathbf{r}_{0}\right)$. Using this relation, $\mathbf{n}_{0}$ and $\mathbf{r}_{0}$ are estimated by minimizing

$$
\sum_{j=1}^{n} \sum_{i=1}^{m}\left|\mathbf{i}^{i j}-\mathbf{i}\left(\mathbf{p}_{h}^{j}, \theta^{i} ; \mathbf{n}_{0}, \mathbf{r}_{0}\right)\right|^{2} .
$$




\section{Experimental Evaluation}

We used laparoscope OTV-S5C (Olympus Optical Co. Ltd., Tokyo, Japan) as an oblique scope for evaluating the proposed camera model and the calibration procedure. The rotary encoder geared to the scope cylinder was used for measuring the rotation angle $\theta$ of the scope cylinder. Its resolution was 0.125 degrees. Polaris (Northern Digital Inc., Ontario, Canada) was used for all other 3D position measurements during the calibration processes. Figure 3 shows the oblique scope with the additional apparatus used in the experiments.

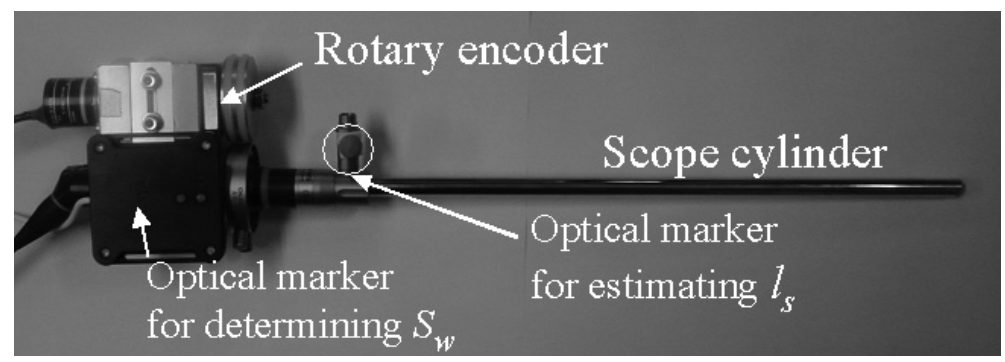

Fig. 3. Oblique-viewing endoscope to which rotary encoder and optical markers are attached.

Images of a checker board pattern were acquired using the oblique scope at different rotation angles of the scope cylinder with the range of $0^{\circ} \leq \theta \leq$ $132^{\circ}$. 3D positions $\mathbf{p}_{h}^{j}$ of the $j$-th crossing points in the checker board in the world coordinate system $S_{w}$ were measured using the Polaris pen probe. 2D coordinates $\mathbf{i}^{i j}$ of projection points corresponding to the $j$-th crossing point were manually specified from the image (whose matrix size was $320 \times 240$ ) acquired at $i$-th rotation angle. Using the above data set for the calibration, $l_{h}\left(\mathbf{n}_{0}\right.$ and $\mathbf{r}_{0}$ ) was estimated. Similarly, other data sets of $\mathbf{p}_{h}^{j}$ and $\mathbf{i}^{i j}$ were acquired for accuracy evaluation of the camera calibration through the different acquisition processes (mostly, at different angles of scope cylinder) from the data sets for the calibration. Using the data sets for evaluation, we defined the projection error $\epsilon$ as

$$
\epsilon=\frac{1}{m n} \sum_{i=1}^{m} \sum_{j=1}^{n}\left|\mathbf{i}^{i j}-\mathbf{i}\left(\mathbf{p}_{h}^{j}, \theta^{i}\right)\right| \quad \text { (pixels) }
$$

where $\mathbf{i}\left(\mathbf{p}_{h}^{j}, \theta^{i}\right)$ denotes computed $2 \mathrm{D}$ coordinates using the calibrated parameters. It should be noted that $\epsilon$ is not residual because the data sets used for the calibration are different from those used for the computation of the error $\epsilon$. The numbers of the angles $\theta^{i}$ were $m=8$ for the calibration data set and $m=7$ for the evaluation data set. The number of manually specified $2 \mathrm{D}$ projection points at each angle was $n=16$ for both data sets. 
We compared the respective errors $\epsilon$ of the following two calibration methods.

- Full calibration: All the processes of the proposed method were applied.

- Partial calibration: $T_{0}$ and $l_{s}$ were estimated, but $l_{h}$ was not. Instead, the $z$-axis of $S_{c}$ was used for the axis for the second rotation.

Figure 4 shows the errors $\epsilon$ for the above two methods when the rotation angle of the scope cylinder $\theta$ varied. The errors $\epsilon$ were constantly low (around 2 pixels) irrespective of $\theta$ in the full calibration while the errors $\epsilon$ were increasingly large as $\theta$ increased in the partial calibration, for example, more than 10 pixels when $\theta$ is greater than $75^{\circ}$. This result shows that the estimation of $l_{h}$, the axis of the second rotation, in the calibration is quite important for accurate calibration.

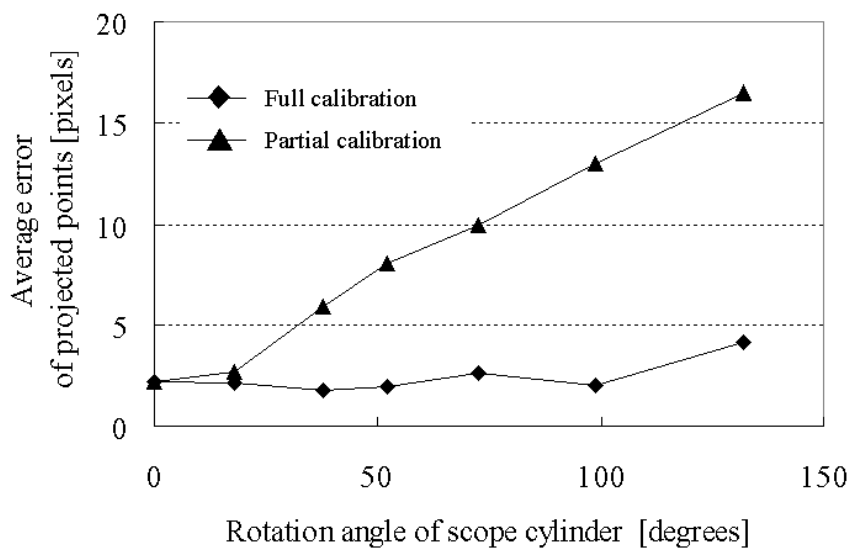

(a)
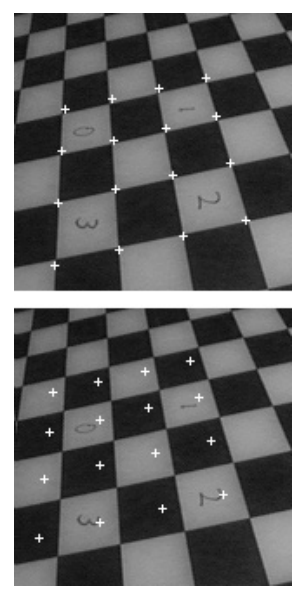

(b)

Fig. 4. Comparisons of projection errors of full and partial calibrations. (a) Projection errors $\epsilon$ (vertical axis) at different rotation angles $\theta$ of scope cylinder (horizontal axis). (b) Computed projection points superimposed onto the original images when $\theta=99^{\circ}$. Top: full calibration, Bottom: partial calibration. The size of each square of the checker board is $14 \times 14 \mathrm{~mm}^{2}$.

\section{Conclusions}

We have developed the first camera model and calibration procedure for the widely used oblique-viewing endoscopes. Both idealized and general models for oblique scopes have been formulated. In the calibration procedure of the general model, Tsai's calibration is performed at zero-rotation of the scope cylinder, and then the variation of the external parameters corresponding to the rotation is prescribed as a function of the rotation angle. The registration error of 2 pixels is attained using our method. Accurate estimation of the rotational axis is included 
in the procedure. The precision of the estimation is proven to exert a significant effect on the overall calibration accuracy especially when the rotation angle is large.

Future work will include the development of rapid and efficient methods for calibration data acquisition as well as the establishment of a more advanced camera model that is able to deal with variations on the internal camera parameters corresponding to the rotation. Using the calibration method proposed in the present paper, we are now developing an augmented reality visualization system combined with intra-operating 3D ultrasound images [10] for laparoscopic procedures using oblique scopes.

Acknowledgement. This work was partly supported by JSPS Research for the Future Program JSPS-RFTF99I00903.

\section{References}

1. Buck SD, et al, A system to support laparoscopic surgery by augmented reality visualization, Lect Notes Comput Sc, vol. 2208 (Proc. MICCAI 2001, Utrecht, The Netherlands), pp. 691-698, 2001.

2. Khadem R, et al, Endoscope calibration and accuracy testing for 3D/2D image registration, Lect Notes Comput Sc, vol. 2208 (Proc. MICCAI 2001, Utrecht, The Netherlands), pp. 1361-1365, 2001.

3. Stefansic JD, et al: Registration of physical space to laparoscopic image space for use in minimally invasive hepatic surgery, IEEE Trans Med Imag, vol. 19, pp. 1012-1023, 2000.

4. Tsai RY, A versatile camera calibration technique for high-accuracy 3D machine vision metrology using off-the-shelf TV cameras and lenses, IEEE Journal of Robotics and Automation, vol. RA-3, pp. 323-344, 1987.

5. Haneishi H, et al., A new method for distortion correction of electronic dendoscope images, IEEE Trans Med Imag, vol. 14, pp. 548-555, 1995.

6. Vijayan K, et al, A new approach for nonlinear distortion correction in endoscopic images based on least squares estimation, IEEE Trans Med Imag, vol. 18, pp. 345-354, 1995.

7. Smith WE, et al, Correction of distortion in endoscopic images, IEEE Trans Med Imag, vol. 11, pp. 117-122, 1992.

8. Shahidi R, et al: Implementation, calibration and accuracy testing of an imageenhanced endoscopy system, IEEE Trans Med Imag, Vol. 21, pp. 1524-1535, 2002.

9. Edwards PJ, et al, Design and evaluation of a system for microscope-assisted guided interventions (MAGI), IEEE Trans Med Imag, vol. 19, pp. 1082-1093, 2000.

10. Nakamoto M, et al, 3D ultrasound system using a magneto-optic hybrid tracker for augmented reality visualization in laparoscopic liver surgery, Lect Notes Comput Sc, vol. 2489 (Proc. MICCAI 2002, Part II, Tokyo, Japan), pp. 148-155, 2002. 